\title{
Harmonic generation in free molecules
}

\author{
D L Andrews \\ School of Chemical Sciences, University of East Anglia, Norwich NR4 7TJ, England
}

Received 19 May 1980

\begin{abstract}
A molecular theory of harmonic generation in free molecules is developed using the principles of quantum electrodynamics. A Cartesian tensor formalism is employed and emphasis is placed on the rotational average which is required to account for the random molecular orientations in the pump beam. It is demonstrated that even harmonics are forbidden to all orders of the multipolar interaction Hamiltonian and in the appendix a new relation involving the rotational average of an arbitrary rank Cartesian tensor is presented.
\end{abstract}

\section{Introduction}

When intense laser light passes through a medium with suitable non-linear optical properties, harmonic frequencies are generated. The most familiar effect is second harmonic generation, or frequency doubling, which is often used as a means of producing UV radiation from lasers operating at visible wavelengths. Although this process can in principle take place in any non-centrosymmetric crystalline solid, it does not generally occur in gases or liquids unless the isotropy is removed by an electric or magnetic field. However, higher order harmonics have been detected in gases, notably the ninth harmonic observed by Grozeva et al (1977) in sodium vapour. By a combination of harmonic generation and frequency mixing processes, coherent radiation of very short XUV wavelengths can thus be produced. Such methods have important applications for the generation and study of ultrashort laser pulses (Auston 1977).

In this study, a molecular theory of harmonic generation in free molecules is developed using the methods of quantum electrodynamics. A Cartesian tensor formalism is employed and particular emphasis is placed on the rotational average which is required to account for the random molecular orientations in the pump beam. Harmonic generation is just one of a large class of non-linear parametric interactions whose observation with circularly polarised light is precluded on symmetry grounds by virtue of this rotational average.

An expression is derived for the intensity of odd harmonics using a new relation concerning the rotational averages. This result gives the explicit dependence of the harmonic intensity on Cartesian components of the non-linear susceptibility tensor. It is then demonstrated that when the full multipolar interaction Hamiltonian is introduced, the exclusion of even harmonics still rigorously applies. 


\section{General theory in the electric dipole approximation}

We start with the quantum electrodynamical Hamiltonian for the system:

$$
H=\sum_{\xi} H_{\text {mol }}(\xi)+\sum_{\xi} H_{\text {int }}(\xi)+H_{\text {rad }}
$$

Here $H_{\text {mol }}(\xi)$ is the usual Schrödinger operator for the molecule labelled $\xi ; H_{\text {int }}(\xi)$ represents the interaction of this molecule with the electromagnetic field, and $H_{\mathrm{rad}}$ is the second-quantised Hamiltonian for the radiation; the sums are taken over all the molecules in the interaction volume. It is convenient to discuss harmonic generation processes within the framework of time-dependent perturbation theory using product eigenstates of $\Sigma_{\xi} H_{\mathrm{mol}}(\xi)$ and $H_{\mathrm{rad}}$ as the basis set and $\Sigma_{\xi} H_{\mathrm{int}}(\xi)$ as the perturbation. In this section, we retain only the leading electric dipole term in the multipole expansion of $H_{\text {int }}(\xi)$; higher order terms are given in $\S 3$. Assuming that there is no static electric or magnetic field applied to the system, we have

$$
H_{\text {int }}(\xi) \simeq-\mu(\xi) \cdot e^{\perp}\left(\boldsymbol{R}_{\xi}\right)
$$

where $\boldsymbol{\mu}(\xi)$ is the electric dipole moment of molecule $\xi$ located at $\boldsymbol{R}_{\xi}$, and $\boldsymbol{e}^{\perp}\left(\boldsymbol{R}_{\xi}\right)$ is the transverse electric field due to the photon flux

$$
\boldsymbol{e}^{\perp}(\boldsymbol{r})=\sum_{\boldsymbol{k}, \lambda}\left(\frac{2 \pi \hbar \omega}{V}\right)^{1 / 2} \mathrm{i}\left(\boldsymbol{e}^{(\lambda)}(\boldsymbol{k}) a^{(\lambda)}(\boldsymbol{k}) \mathrm{e}^{\mathrm{i} \boldsymbol{k} \cdot \boldsymbol{r}}-\overline{\boldsymbol{e}}^{-(\lambda)}(\boldsymbol{k}) a^{\dagger(\lambda)}(\boldsymbol{k}) \mathrm{e}^{-\mathrm{i} \boldsymbol{k} \cdot \boldsymbol{r}}\right) .
$$

In equation $(3), a^{(\lambda)}(\boldsymbol{k})$ and $a^{\dagger(\lambda)}(\boldsymbol{k})$ are the annihilation and creation operators for photons of wave-vector $\boldsymbol{k}$ (circular frequency $\omega=c|\boldsymbol{k}|$ ) and polarisation vector $\boldsymbol{e}^{(\lambda)} ; \boldsymbol{V}$ is the quantisation volume.

The detailed temporal evolution of a harmonic generated by a pulse of laser light can be studied using quantum electrodynamical methods described in other recent papers by Andrews (1978) and Elgin (1980). For simplicity, however, we shall now consider the continuous-wave solution with a time-independent rate given by the Fermi rule

$$
\Gamma=\frac{2 \pi}{\hbar}\left|\mathcal{M}_{f i}\right|^{2} \rho_{f}
$$

here $\mathscr{M}_{f i}$ is the matrix element connecting the initial and final states of the system, and $\rho_{f}$ is the density of final states of the radiation field. In the dilute gas approximation, $\mathcal{M}_{f i}$ may be written as a sum of contributions $M_{f i}$ from each molecule,

$$
\mathscr{M}_{f i}=\sum_{\xi} M_{f i}^{\xi}
$$

with the usual perturbation series expansion

$$
\begin{aligned}
M_{f i}^{\xi}=\left\langle f_{\mathrm{s}}^{\xi}\left|H_{\mathrm{int}}(\xi)\right| i_{\mathrm{s}}^{\xi}\right\rangle & +\sum_{I_{\mathrm{s}}^{\prime}} \frac{\left\langle f_{\mathrm{s}}^{\xi}\left|H_{\mathrm{int}}(\xi)\right| I_{\mathrm{s}}^{\xi}\right\rangle\left\langle I_{\mathrm{s}}^{\xi}\left|H_{\mathrm{int}}(\xi)\right| i_{\mathrm{s}}^{\xi}\right\rangle}{E\left(i_{\mathrm{s}}^{\xi}\right)-E\left(I_{\mathrm{s}}^{\xi}\right)} \\
& +\sum_{I_{\mathrm{s}}, I I_{\mathrm{s}}} \frac{\left\langle f_{\mathrm{s}}^{\xi}\left|H_{\mathrm{int}}(\xi)\right| I I_{\mathrm{s}}^{\xi}\right\rangle\left\langle I I_{\mathrm{s}}^{\xi}\left|H_{\mathrm{int}}(\xi)\right| I_{\mathrm{s}}^{\xi}\right\rangle\left\langle I_{\mathrm{s}}^{\xi}\left|H_{\mathrm{int}}(\xi)\right| i_{\mathrm{s}}^{\xi}\right\rangle}{\left(E\left(i_{\mathrm{s}}^{\xi}\right)-E\left(I I_{\mathrm{s}}^{\xi}\right)\right)\left(E\left(i_{\mathrm{s}}^{\xi}\right)-E\left(I_{\mathrm{s}}^{\xi}\right)\right)}+\ldots
\end{aligned}
$$

In this equation states and energies, for example $\left|i_{\mathrm{s}}^{\xi}\right\rangle$ and $E\left(i_{\mathrm{s}}^{\xi}\right)$, relate to the system comprising the molecule $\xi$ plus the radiation-hence the introduction of the subscript $s$. The primes denote omission from the intermediate-state summations of the initial and final states of the system. 
Thus the leading contribution to equation (6) for phase-matched $m$-harmonic emission in the forward direction is as follows (using the implied summation convention for repeated indices):

$$
M_{f i}^{\xi}=(-\mathrm{i})^{m-1}\left(\frac{2 \pi \hbar \omega}{V}\right)^{(m+1) / 2}\left(\frac{n ! m}{(n-m) !}\right)^{1 / 2} T_{i_{1} \ldots i_{m+1}}^{\xi} e_{i_{1}} \ldots e_{i_{m}} \bar{e}_{i_{m+1}}^{\prime}
$$

Here $n$ is the number of photons in the incident radiation mode with frequency $\omega$ and polarisation vector $\boldsymbol{e}$; it is assumed that the radiation mode for the harmonic, with polarisation $e^{\prime}$, is initially empty. The non-linear polarisability tensor $T_{i_{1} \ldots i_{m+1}}^{\xi_{m}}$ can be evaluated with the aid of time-ordered diagrams (Ward 1965), a typical example of which is shown in figure 1. This diagram represents the successive absorption of $q$ photons, followed by emission of a photon at the $m$-harmonic frequency, followed by further $(m-q)$ absorptions before the molecule returns to its ground state. There are $(m+1)$ topologically distinct diagrams to be considered, each of which we can label with an index $q$ in the range $0 \leqslant q \leqslant m$. The full result for the susceptibility tensor may be expressed most compactly in the following form, dropping the label $\xi$ for clarity:

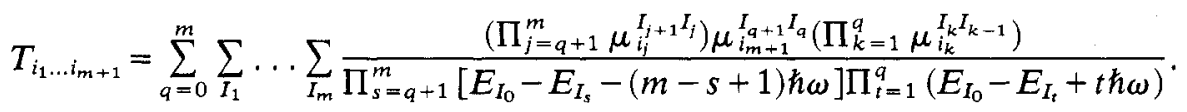

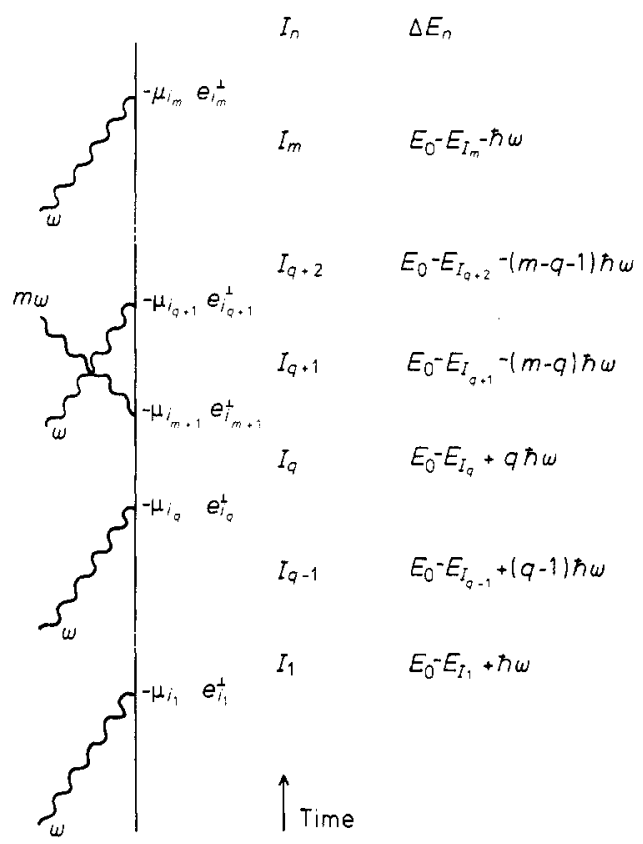

Figure 1. One of the time-ordered diagrams for $m$-harmonic generation. $I_{n}$ is the label for successive intermediate states of the molecule and $\Delta E_{n}$ is the corresponding difference in energy of the system from the initial state.

Here $\boldsymbol{\mu}^{I_{a} I_{b}}$ is the electric dipole transition moment for the transition between-intermediate molecular states $\left|I_{a}\right\rangle \leftarrow\left|I_{b}\right\rangle$. The energy of state $\left|I_{a}\right\rangle$ is $E_{I_{a}}$, and both $\left|I_{0}\right\rangle$ and $\left|I_{m+1}\right\rangle$ are identified with the ground state $|0\rangle$ of the molecule. In writing equation (8), use has been made of the convention

$$
\prod_{l=p}^{p-1} f_{l}(l)=1 .
$$


Now from equations (4), (5) and (7) the rate of harmonic emission is given by

$$
\Gamma=\left(\frac{2 \pi \hbar \omega}{V}\right)^{m+1} \frac{2 \pi m n ! \rho_{f}}{(n-m) ! \hbar}\left|\sum_{\xi} T_{i_{1} \ldots i_{m+1}}^{\xi} e_{i_{1}} \ldots e_{i_{m}} \bar{e}_{i_{m+1}}^{\prime}\right|^{2} .
$$

The expression is strictly applicable only in the case where the incident radiation is represented by a pure number state. The coherence and statistical properties of the light may be taken into account by adopting a more general representation in terms of a linear combination of such number states, and the corresponding result for the harmonic radiant intensity $\mathscr{I}^{(m)}$ may then be written in terms of the mean irradiance $\bar{I}_{0}$ and the degree of $m$ th order coherence $g^{(m)}$ as follows:

$$
\mathscr{F}^{(m)}=\left(\frac{2 \pi}{c}\right)^{m-1}\left(\frac{m \omega}{c}\right)^{4} g^{(m)} \bar{I}_{0}^{m}\left|\sum_{\xi} T_{i_{1} \ldots i_{m+1}}^{\xi} e_{i_{1}} \ldots e_{i_{m}} \bar{e}_{i_{m+1}}^{\prime}\right|^{2} .
$$

For harmonic generation in free molecules, this expression has to be averaged over all possible molecular orientations. Denoting the required rotational average for molecule $\xi$ by angular brackets $\langle\ldots\rangle_{\xi}$, we thus have

$$
\begin{aligned}
\mathscr{I}^{(m)}=\left(\frac{2 \pi}{c}\right)^{m-1} & \left(\frac{m \omega}{c}\right)^{4} g^{(m)} \bar{I}_{0}^{m} \\
& \times\left(\sum_{\xi}\left\langle T_{i_{1} \ldots i_{m+1}}^{\xi} \bar{T}_{i_{m+2} \ldots i_{2 m+2}}^{\xi} e_{i_{1}} \ldots e_{i_{m}} \bar{e}_{i_{m+1}}^{\prime} \bar{e}_{i_{m+2}} \ldots \bar{e}_{i_{2 m+1}} e_{i_{2 m+2}}^{\prime}\right\rangle_{\xi}\right. \\
& \left.+\sum_{\xi^{\prime} \neq \xi}\left\langle\bar{T}_{i_{m+2} \ldots i_{2 m+2}}^{\xi} \bar{e}_{i_{m+2}} \ldots \bar{e}_{i_{2 m+1}} e_{i_{2 m+2}}^{\prime}\right\rangle_{\xi^{\prime}} \sum_{\xi}\left\langle T_{i_{1} \ldots i_{m+1}}^{\xi} e_{i_{1}} \ldots e_{i_{m}} \bar{e}_{i_{m+1}}^{\prime}\right\rangle_{\xi}\right)
\end{aligned}
$$

assuming there is no orientational correlation between different molecules. The result of rotationally averaging each of the terms in angular brackets is then independent of the particular molecule upon which the average is performed. Hence with $\mathcal{N}$ molecules in the interaction volume we have

$$
\begin{aligned}
\mathscr{I}^{(m)}=\left(\frac{2 \pi}{c}\right)^{m-1} & \left(\frac{m \omega}{c}\right)^{4} g^{(m)} \bar{I}_{0}^{m} \\
& \times\left(\mathcal{N}\left(T_{i_{1} \ldots i_{m+1}} \bar{T}_{i_{m+2} \ldots i_{2 m+2}} e_{i_{1}} \ldots e_{i_{m}} \bar{e}_{i_{m+1}}^{\prime} \bar{e}_{i_{m+2}} \ldots \bar{e}_{i_{2 m+1}} e_{i_{2 m+2}}^{\prime}\right\rangle\right. \\
& \left.+\mathcal{N}(\mathcal{N}-1)\left|\left\langle T_{i_{1} \ldots i_{m+1}} e_{i_{1}} \ldots e_{i_{m}} \bar{e}_{i_{m+1}}^{\prime}\right)\right|^{2}\right) .
\end{aligned}
$$

The two terms in the angular brackets respectively denote incoherent and coherent contributions to the harmonic intensity. The incoherent contribution is relatively weak and is only significant when considering non-forward harmonic emission; see for example Kielich and Kozierowski (1972). For present purposes we may ignore this term. Hence we may write

$$
\mathscr{I}^{(m)} \simeq \mathscr{L}^{(m)}\left|\left\langle T_{i_{1} \ldots i_{m+1}} e_{i_{1}} \ldots e_{i_{m}} \bar{e}_{i_{m+1}}^{\prime}\right\rangle\right|^{2}
$$

where

$$
\mathscr{L}^{(m)}=\left(\frac{2 \pi}{c}\right)^{m-1}\left(\frac{m \omega}{c}\right)^{4} g^{(m)} \bar{I}_{0}^{m} \mathcal{N}^{2} .
$$

The detailed procedure for dealing with the rotational average of a Cartesian tensor product, as in equation (14), has been discussed in a previous paper (Andrews and 
Thirunamachandran 1977). The result may be written as

$$
\mathscr{I}^{(m)}=\mathscr{L}^{(m)}\left|T_{\lambda_{1} \ldots \lambda_{m+1}+1} e_{i_{1}} \ldots e_{i_{m}} \bar{e}_{i_{m+1}}^{\prime} I_{i_{1} \ldots i_{m+1} ; \lambda_{1} \ldots \lambda_{m+1}}^{(m+1)}\right|^{2} .
$$

Here components of the susceptibility tensor are referred to a molecule-fixed frame and components of the polarisation vectors are referred to a space-fixed frame; $I_{i_{1} \ldots i_{m+1} ; \lambda_{1} \ldots \lambda_{m+1}}^{(m+1)}$ is the rotational average of a product of direction cosines $l_{i_{p} \lambda_{p}}$ relating coordinates in the two frames and defined by

$I_{i_{1} \ldots i_{m+1} ; \lambda_{1} \ldots \lambda_{m+1}}^{(m+1)}=\frac{1}{8 \pi^{2}} \int_{0}^{2 \pi} \int_{0}^{\pi} \int_{0}^{2 \pi} \prod_{p=1}^{m+1} l_{i_{p} \lambda_{p}}(\phi, \theta, \psi) \sin \theta \mathrm{d} \phi \mathrm{d} \theta \mathrm{d} \psi$

where $\phi, \theta$ and $\psi$ are the Euler angles for the frame transformation.

The result for the rotational average can be written explicitly as a linear combination of isotropic tensor products; in general we have

$$
I_{i_{1} \ldots i_{n} ; \lambda_{1} \ldots \lambda_{n}}^{(n)}=\sum_{r, s} m_{r s}^{(n)} f_{i_{1} \ldots i_{n}}^{(n ; r)} g_{\lambda_{1} \ldots \lambda_{n}}^{(n ; s)}
$$

In this equation $f_{i_{1} \ldots i_{n}}^{(n ; r)}$ are isotropic tensor components referred to the space-fixed frame, $g_{\lambda_{1} \ldots \lambda_{n}}^{(n ; s)}$ are the corresponding tensor components referred to the molecule-fixed frame, and $m_{r s}^{(n)}$ are real coefficients which can be determined by matrix inversion methods (Andrews and Thirunamachandran 1977). From equations (16) and (18) we thus have

$$
\mathscr{I}^{(m)}=\mathscr{L}^{(m)}\left|\sum_{r, s} m_{r s}^{(m+1)}\left(e_{i_{1}} \ldots e_{i_{m}} \bar{e}_{i_{m+1}}^{\prime} f_{i_{1} \ldots i_{m+1}}^{(m+1 ; r)}\right)\left(T_{\lambda_{1} \ldots \lambda_{m+1}} g_{\lambda_{1} \ldots \lambda_{m+1}}^{(m+1 ; s)}\right)\right|^{2} .
$$

In this expression the product of polarisation vector components $e_{i_{1}} \ldots e_{i_{m}} \bar{e}_{i_{m+1}}^{\prime}$, whica we may refer to as the polarisation tensor, is contracted with each isotropic tensor $f_{i_{1} \ldots i_{m+1}}^{(m+1 ; r)}$ in the space-fixed frame, and the non-linear susceptibility tensor $T_{\lambda_{1} \ldots \lambda_{m+1}}$ is contracted with each isotropic tensor $g_{\lambda_{1} \ldots \lambda_{m+1}}^{(m+1 ; s)}$ in the molecular frame.

For even $n$, isotropic tensors of rank $n$ are products of $n / 2$ Kronecker delta tensors such as $\delta_{i_{1} i_{2}} \ldots \delta_{i_{n-1} i_{n}}$; for odd $n$ they are products of one Levi-Civita antisymmetric tensor and $(n-3) / 2$ Kronecker deltas, for example $\epsilon_{i_{1} i_{2} i_{3}} \delta_{i_{4} i_{5}} \ldots \delta_{i_{n-1} i_{n}}$. Because of the form which these tensors take, it is immediately possible to draw some general conclusions regarding the result of equation (19), using the fact that the polarisation tensor $e_{i_{1}} \ldots e_{i_{m}} \bar{e}_{i_{m+1}}^{\prime}$ is symmetric with respect to any permutation of the indices $i_{1} \ldots i_{m}$. From this it is clear that the result of every index contraction $\left(e_{i_{1}} \ldots e_{i_{m}} \bar{e}_{i_{m+1}}^{\prime} f_{i_{1} \ldots i_{m+1}}^{(m+r)}\right)$ is zero if $m+1$ is odd, for then a Levi-Civita tensor would appear with an antisymmetry in two or three of the indices $i_{1} \ldots i_{m}$. Hence the coherent generation of even $m$ harmonics in isotropic media is forbidden, as is well known. This conclusion is naturally valid irrespective of the molecular geometry and it is shown in $\S 3$ that the validity is retained even when all the higher order multipolar contributions to the interaction Hamiltonian are taken into account.

For the generation of odd $m$ harmonics, it is important to note that the contraction $\left(e_{i_{1}} \ldots e_{i_{m}} \bar{e}_{i_{m+1}}^{\prime} f_{i_{1} \ldots i_{m+1}}^{(m+r)}\right)$ must contain at least one factor $(\boldsymbol{e}, \boldsymbol{e})$ and this is zero for circularly polarised light. Hence we also directly obtain the result that generation of any harmonic is forbidden if the pump beam is circularly polarised; see also Bloembergen (1969), Tang and Rabin (1971). It should be added, however, that these arguments are only applicable to the coherent contribution to the harmonic intensity. The usually insignificant contribution from the incoherent term in equation (13) remains finite both 
for even harmonics and for circularly polarised radiation, whether for forward or non-forward emission.

In passing, it is worth noting that the same reasoning can be applied to other non-linear interactions in isotropic media. In general, the result always involves a linear combination of scalars formed by contracting the polarisation tensor, which is the product of polarisation vector components for each photon involved in the process, with an isotropic tensor of the same rank. Therefore, unless the polarisation tensor has a zero-weight component (corresponding in angular momentum terms to a $\Delta m=0$ process), all such scalars vanish identically and the process is forbidden. The conditions which this imposes on the polarisation tensor are quite restrictive and, in general, we can conclude that every parametric process which involves the absorption or emission of two or more circularly polarised photons of the same handedness and direction of propagation is forbidden in an isotropic medium. This is true regardless of whether or not the photons have the same frequency. In the case where there is one absorption and one emission of a circularly polarised photon, the process is forbidden if the photons have the same direction of propagation but opposite handedness.

Returning to equation (19) and assuming in the light of the above remarks that the incident beam is plane polarised, we find that the contraction of $e_{i_{1}} \ldots e_{i_{m}} \bar{e}_{i_{m+1}}^{\prime}$ with any $f_{i_{1} \ldots i_{m+1}}^{(m+r)}$ gives the result $\left(\boldsymbol{e}, \boldsymbol{e}^{\prime}\right)$. Hence, as for the trivial case of forward Rayleigh scattering $(m=1)$, there is retention of polarisation in the harmonic emission. We now introduce a fully index-symmetric tensor $\tilde{T}_{\lambda_{1} \ldots \lambda_{m+1}}$, derived from $T_{\lambda_{1} \ldots \lambda_{m+1}}$ by summing over all index permutations and multiplying by a normalising factor $1 /(m+1)$ !. With $\boldsymbol{e}=\boldsymbol{e}^{\prime}$, equation (19) can then be written as

$$
\mathscr{I}^{(m)}=\mathscr{L}^{(m)}\left|\sum_{r, s} m_{r s}^{(m+1)}\left(e_{i_{1}} \ldots e_{i_{m+1}} f_{i_{1} \ldots i_{m+1}}^{(m+1 ; r)}\right)\left(\tilde{T}_{\lambda_{1} \ldots \lambda_{m+1}} g_{\lambda_{1} \ldots \lambda_{m+1}}^{(m+1 ; s)}\right)\right|^{2} .
$$

Note that this expression is exact and not an approximation based on the Kleinman condition of negligible dispersion (Kleinman 1962). The introduction of $\tilde{T}_{\lambda_{1} \ldots \lambda_{m+1}}$ is simply a reflection of the fact that any antisymmetric components of the susceptibility tensor give vanishing contributions to $\mathscr{I}^{(m)}$.

Now we have

$$
e_{i_{1}} \ldots e_{i_{m+1}} f_{i_{1} \ldots i_{m+1}}^{(m+1 ; r)}=1
$$

for all $r$, and by virtue of the index symmetry of $\tilde{T}_{\lambda_{1} \ldots \lambda_{m+1}}$ we also have

$$
\tilde{T}_{\lambda_{1} \ldots \lambda_{m+1}} g_{\lambda_{1} \ldots \lambda_{m+1}}^{(m+1 ; s)}=\chi^{(m)}
$$

for all $s$, where

$$
\chi^{(m)}=\tilde{T}_{\lambda_{1} \lambda_{1} \lambda_{3} \lambda_{3} \ldots \lambda_{m} \lambda_{m}}
$$

Hence, equation (20) may be written as

$$
\mathscr{I}^{(m)}=\mathscr{L}^{(m)}\left|\chi^{(m)}\right|^{2}\left(\sum_{r, s} m_{r s}^{(m+1)}\right)^{2} .
$$

Final evaluation of this expression thus requires knowledge of the rotational average coefficients $m_{r s}^{(m+1)}$ which are known only for $m \leqslant 7$ (Andrews and Thirunamachandran 1977). Even for seventh-harmonic generation requiring the rank-8 average, the procedure for determining all the $m_{r s}^{(8)}$ necessitates the construction and inversion of a 
$91 \times 91$ matrix and the problem rapidly becomes more lengthy with increasing $m$. However, it is possible to obtain a general result for $\Sigma_{r, s} m_{r s}^{(m+1)}$ by a method described in the appendix and using this result we obtain the final expression for the radiant intensity of an odd $m$ harmonic:

$$
\mathscr{I}^{(m)}=\frac{1}{m+2}\left(\frac{2 \pi}{c}\right)^{m-1}\left(\frac{m \omega}{c}\right)^{4} g^{(m)} \bar{I}_{0}^{m} \mathcal{N}^{2}\left|\chi^{(m)}\right|^{2} .
$$

\section{Consideration of higher order terms in the interaction Hamiltonian for coherent generation of even harmonics}

We can now show that even harmonics are forbidden not only in the electric dipole approximation, but also when all higher order multipolar contributions are considered. The methods used here are similar to those adopted by Healy (1974) in a study of optical rotation; the symmetry arguments used in this section, however, relate to the properties of the radiation field rather than the molecular response tensors.

The full expression for the interaction Hamiltonian $H_{\text {int }}(\xi)$ before carrying out a multipolar decomposition can be written as follows:

$H_{\mathrm{int}}(\xi)=-\int \boldsymbol{P}^{(\xi) \perp}(\boldsymbol{r}) \cdot e^{\perp}(\boldsymbol{r}) \mathrm{d}^{3} \boldsymbol{r}-\int \boldsymbol{M}^{(\xi)}(\boldsymbol{r}) \cdot \boldsymbol{b}(\boldsymbol{r}) \mathrm{d}^{3} \boldsymbol{r}+\frac{1}{2} \iint O_{i j}^{(\xi)}\left(\boldsymbol{r}, \boldsymbol{r}^{\prime}\right) b_{i}(\boldsymbol{r}) b_{j}\left(\boldsymbol{r}^{\prime}\right) \mathrm{d}^{3} \boldsymbol{r} \mathrm{d}^{3} \boldsymbol{r}^{\prime}$

see for example Babiker et al (1974), Power and Thirunamachandran (1980). Here $\boldsymbol{P}^{(\xi) \perp}(\boldsymbol{r})$ is the transverse electric polarisation vector field, $\boldsymbol{M}^{(\xi)}(\boldsymbol{r})$ is the magnetisation vector field and $\mathbf{O}^{(\xi)}\left(\boldsymbol{r}, \boldsymbol{r}^{\prime}\right)$ the diamagnetisation tensor associated with molecule $\xi ; \boldsymbol{e}^{\perp}(\boldsymbol{r})$ is the transverse electric field and $\boldsymbol{b}(\boldsymbol{r})$ the magnetic field resulting from the photon flux. The multipolar expressions for $\boldsymbol{P}^{(\xi) \perp}(\boldsymbol{r}), \boldsymbol{M}^{(\xi)}(\boldsymbol{r})$ and $\mathbf{O}^{(\xi)}\left(\boldsymbol{r}, \boldsymbol{r}^{\prime}\right)$ may be derived from the following equations by expanding the delta functions in Taylor series about $\left(\boldsymbol{r}-\boldsymbol{R}_{\xi}\right)$ :

$$
\begin{aligned}
& \boldsymbol{P}^{(\xi) \perp}(\boldsymbol{r})=\sum_{\boldsymbol{x}^{\prime}(\xi)} e_{\alpha(\xi)}\left(\boldsymbol{q}_{\alpha(\xi)}-\boldsymbol{R}_{\xi}\right) \int_{0}^{1} \delta^{\perp}\left[\boldsymbol{r}-\boldsymbol{R}_{\xi}-\lambda\left(\boldsymbol{q}_{\alpha(\xi)}-\boldsymbol{R}_{\xi}\right)\right] \mathrm{d} \lambda \\
& \boldsymbol{M}^{(\xi)}(\boldsymbol{r})=\sum_{\alpha(\xi)} \frac{e_{\alpha(\xi)}}{2 m_{\alpha(\xi)} \mathcal{C}}\left(\left[\left(\boldsymbol{q}_{\alpha(\xi)}-\boldsymbol{R}_{\xi}\right) \times \boldsymbol{p}_{\alpha(\xi)}\right] \int_{0}^{1} \lambda \delta\left[\boldsymbol{r}-\boldsymbol{R}_{\xi}-\lambda\left(\boldsymbol{q}_{\alpha(\xi)}-\boldsymbol{R}_{\xi}\right)\right] \mathrm{d} \lambda\right. \\
& \left.+\int_{0}^{1} \lambda \delta\left[r-\boldsymbol{R}_{\xi}-\lambda\left(\boldsymbol{q}_{\alpha(\xi)}-\boldsymbol{R}_{\xi}\right)\right] \mathrm{d} \lambda\left[\left(\boldsymbol{q}_{\alpha(\xi)}-\boldsymbol{R}_{\xi}\right) \times \boldsymbol{p}_{\alpha(\xi)}\right]\right) \\
& O_{i j}^{(\xi)}\left(\boldsymbol{r}, \boldsymbol{r}^{\prime}\right)=\sum_{\alpha(\xi)} \frac{e_{\alpha(\xi)}^{2}}{m_{\alpha(\xi)} c^{2}}\left[\left(\boldsymbol{q}_{\alpha(\xi)}-\boldsymbol{R}_{\xi}\right)^{2} \delta_{i j}-\left(\boldsymbol{q}_{\alpha(\xi)}-\boldsymbol{R}_{\xi}\right)_{i}\left(\boldsymbol{q}_{\alpha(\xi)}-\boldsymbol{R}_{\xi}\right)_{j}\right] \\
& \times \int_{0}^{1} \int_{0}^{1} \lambda \lambda^{\prime} \delta\left[\boldsymbol{r}-\boldsymbol{R}_{\xi}-\lambda\left(\boldsymbol{q}_{\alpha(\xi)}-\boldsymbol{R}_{\xi}\right)\right] \delta\left[\boldsymbol{r}^{\prime}-\boldsymbol{R}_{\xi}-\lambda^{\prime}\left(\boldsymbol{q}_{\alpha(\xi)}-\boldsymbol{R}_{\xi}\right)\right] \mathrm{d} \lambda \mathrm{d} \lambda^{\prime} .
\end{aligned}
$$

In each case the sum is taken over all particles $\alpha(\xi)$ of charge $e_{\alpha(\xi)}$ and mass $m_{\alpha(\xi)}$ in the molecule $\xi$.

The matrix element $M_{f i}^{\xi}$ for the harmonic generation process may be written as a sum of terms in which each photon absorption or emission is associated with one of the 
multipolar contributions to (26). It is readily shown that each term takes the form

$$
\begin{aligned}
(-\mathrm{i})^{m-p+q-1} & m^{q}\left(\frac{2 \pi \hbar \omega}{V}\right)^{(m+1) / 2}\left(\frac{\omega}{c}\right)^{p+q}\left(\frac{n ! m}{(n-m) !}\right)^{1 / 2} \\
& \times \Lambda_{i_{1} \ldots i_{m+p+q+1}} w_{i_{1}}^{(1)} \ldots w_{i_{m+1}}^{(m+1)} \hat{k}_{i_{m+2}} \ldots \hat{k}_{i_{m+p+q+1}} .
\end{aligned}
$$

Here $\Lambda_{i_{1} \ldots i_{m+p+q+1}}$ is a molecular response tensor of rank $(m+p+q+1)$, and $\boldsymbol{w}^{(n)}$ are unit vectors (either real or complex) in the plane perpendicular to the incident and harmonic propagation unit vectors $\hat{k}$. In the electric dipole approximation of $\S 2$, both $p$ and $q$ are zero, cf equation (7).

Because harmonic generation is a coherent process, the result for the harmonic intensity involves the square modulus of the rotational average of $M_{f i}^{\xi}$ (for incoherent processes the average of $\left|M_{f i}^{\xi}\right|^{2}$ is required). Hence, we have to evaluate

$$
\begin{aligned}
\left|\Lambda_{\lambda_{1} \ldots \lambda_{m+p+q+1}} w_{i_{1}}^{(1)} \ldots w_{i_{m+1}}^{(m+1)} \hat{k}_{i_{m+2}} \ldots \hat{k}_{i_{m+p+q+1}} I_{i_{1} \ldots i_{m+p+q+1} ; \lambda_{i} \ldots \lambda_{m+p+q+1}}^{(m+p+q+1)}\right|^{2} \\
=\mid \sum_{r, s} m_{r s}^{(m+p+q+1)}\left(w_{i_{1}}^{(1)} \ldots w_{i_{m+1}}^{(m+1)} \hat{k}_{i_{m+2}} \ldots \hat{k}_{i_{m+p+q+1}} f_{i_{1} \ldots i_{m+p+q+1}}^{(m+p+q+1 ; r)}\right) \\
\quad \times\left.\left(\Lambda_{\lambda_{1} \ldots \lambda_{m+p+q+1}} g_{\lambda_{1} \ldots \lambda_{m+p+q}}^{(m+p+q+1 ; s)}\right)\right|^{2}
\end{aligned}
$$

We now consider the two cases where $p+q$ is either even or odd.

Suppose $p+q$ is even. For an even $m$ harmonic, $m+p+q+1$ is odd and the isotropic tensors $f_{i_{1} \ldots i_{m}+p+q+1}^{(m+p+q+1)}$ involve one Levi-Civita tensor and $(m+p+q-2) / 2$ Kronecker deltas. The Levi-Civita tensor must contract with two non-collinear $w$ vectors and one $\hat{\boldsymbol{k}}$ vector to give a non-vanishing result; hence there remain $(m-1) \boldsymbol{w}$ vectors and $(p+q-1) \hat{\boldsymbol{k}}$ vectors to contract with the Kronecker deltas. Since $(p+q-1)$ is odd, the result must involve at least one scalar product between a $\boldsymbol{w}$ vector and a $\hat{\boldsymbol{k}}$ vector; therefore, the result vanishes entirely.

If $p+q$ is odd and $m$ is even, then $(m+p+q+1)$ is even and $f_{i_{1} \ldots i_{m+p+q+1}}^{(m+p+q+1)}$ is a product of $(m+p+q+1) / 2$ Kronecker deltas. The contraction with the product of $w$ and $\hat{k}$ vectors in equation (31) thus again involves at least one scalar product between a $w$ vector and a $\hat{\boldsymbol{k}}$ vector, leading to a vanishing result.

Hence, to all orders in the multipole expansion of the interaction Hamiltonian, coherent generation of even harmonics is forbidden in free molecules.

\section{Acknowledgment}

I acknowledge with thanks helpful comments from Dr T Thirunamachandran.

\section{Appendix. Proof of the relation $\Sigma_{r, s} m_{r s}^{(2 n)}=1 /(2 n+1)$}

Consider the rotational average of $\mathrm{e}^{\mathrm{i} \boldsymbol{A} \cdot \boldsymbol{a}}$, where $\boldsymbol{A}$ is a real molecule-fixed vector and $\boldsymbol{a}$ is a real space-fixed vector; by explicit integration using the Euler angle rotation matrix it is readily shown that

$$
\left\langle\mathrm{e}^{\mathrm{i} \boldsymbol{A} \cdot \boldsymbol{a}}\right\rangle=\frac{\sin A a}{A a} .
$$


Taking the Taylor series expansions of both sides of (A.1) we obtain

$$
\sum_{p=0}^{\infty} \frac{\left\langle(\mathbf{i} \boldsymbol{A} \cdot \boldsymbol{a})^{p}\right\rangle}{p !}=\sum_{q=0}^{\infty} \frac{(\mathrm{i} A a)^{2 q}}{(2 q+1) !} .
$$

The left-hand side of (A.2) can be written as

$$
\begin{aligned}
\sum_{p=0}^{\infty} \frac{\left\langle(\mathrm{i} \boldsymbol{A} \cdot \boldsymbol{a})^{p}\right\rangle}{p !} & =\sum_{p=0}^{\infty} \frac{\mathrm{i}^{p}}{p !}\left\langle A_{i_{1}} \ldots A_{i_{p}} a_{i_{1}} \ldots a_{i_{p}}\right\rangle \\
& =\sum_{p=0}^{\infty} \frac{\mathrm{i}^{p}}{p !} A_{\lambda_{1}} \ldots A_{\lambda_{p}} a_{i_{1}} \ldots a_{i_{p}} I_{i_{1} \ldots i_{p} ; \lambda_{1} \ldots \lambda_{p}}^{(p)} \\
& =\sum_{p=0}^{\infty} \frac{\mathrm{i}^{p}}{p !} \sum_{r, s} m_{r s}^{(p)}\left(a_{i_{1}} \ldots a_{i_{p}} f_{i_{1} \ldots i_{p}}^{(p ; r)}\right)\left(A_{\lambda_{1}} \ldots A_{\lambda_{p}} g_{\lambda_{1} \ldots \lambda_{p}}^{(p ; s)}\right) .
\end{aligned}
$$

Now

$$
\begin{aligned}
a_{i_{1}} \ldots a_{i_{p}} f_{i_{1} \ldots i_{p}}^{(p ; r)} & = \begin{cases}a^{p} & (p \text { even }, \text { all } r) \\
0 & (p \text { odd }, \text { all } r)\end{cases} \\
A_{\lambda_{1}} \ldots A_{\lambda_{p}} g_{\lambda_{1} \ldots \lambda_{p}}^{(p ; s)} & = \begin{cases}A^{p} & (p \text { even, all } s) \\
0 & (p \text { odd }, \text { all } s)\end{cases}
\end{aligned}
$$

in view of the form of the isotropic tensors (see main text). Hence, we have

$$
\sum_{p=0}^{\infty} \frac{\left\langle(\mathbf{i} \boldsymbol{A} \cdot \boldsymbol{a})^{p}\right\rangle}{p !}=\sum_{m=0}^{\infty} \frac{(\mathrm{i} \boldsymbol{A} a)^{2 m}}{(2 m) !} \sum_{r, s} m_{r s}^{(2 m)} .
$$

Comparing coefficients of $(\mathrm{i} A a)^{2 n}$ on the right-hand sides of equations (A.2) and (A.6) hence gives the result

$$
\sum_{r, s} m_{r s}^{(2 n)}=\frac{1}{2 n+1} .
$$

This relation will provide a useful check on results for high rank rotational averages with $2 n>8$, which have yet to be calculated. The condition represented by (A.7) must be satisfied by both reducible and irreducible forms of these averages.

\section{References}

Andrews D L 1978 J. Phys. B: Atom. Molec. Phys. 112655

Andrews D L and Thirunamachandran T $1977 \mathrm{~J}$. Chem. Phys. 675026

Auston D H 1977 Ultrashort Light Pulses-Picosecond Techniques and Applications ed S L Shapiro (Berlin: Springer) p 123

Babiker M, Power E A and Thirunamachandran T 1974 Proc. R. Soc. A 338235

Bloembergen N 1969 Polarisation Matière et Rayonnement ed Société Française de Physique (Paris: Presses Universitaires de France) p 109

Elgin J N $1980 J$. Phys. B: Atom. Molec. Phys. 13655

Grozeva M G, Metchkov D I, Mitev V M, Pavlov L I and Stamenov K V 1977 Opt. Commun. 2377

Healy W P 1974 J. Phys. B: Atom. Molec. Phys. 71633

Kielich S and Kozierowski M 1972 Opt. Commun. 4395

Kleinman D A 1962 Phys. Rev. 1261977

Power E A and Thirunamachandran T 1980 Proc. R. Soc. A 372265

Tang C L and Rabin H 1971 Phys. Rev. B 124025

Ward J F 1965 Rev. Mod. Phys. 371 